\title{
Supernumerary Cranial Sutures
}

National Cancer Institute

\section{Source}

National Cancer Institute. Supernumerary Cranial Sutures. NCI Thesaurus. Code

C124642.

Sutures commonly found within the parietal and occipital bones of the skull due to their multiple ossification centers. These sutures exhibit a zigzag pattern with interdigitations and sclerotic borders similar to, and join and merge with the major sutures. 